\title{
Molecular identification of intergenus crosses involving catfish hybrids: risks for aquaculture production
}

\author{
Diogo T. Hashimoto 1 , Fernanda D. Prado ${ }^{2}$, Fausto Foresti ${ }^{3}$ and Fábio Porto-Foresti ${ }^{2}$
}

\begin{abstract}
Monitoring of the interspecific hybrid production and trade is essential for the appropriate management of these animals in fish farms. The identification of catfish hybrids by morphological analysis is unreliable, particularly of juveniles and post-F1 individuals. Therefore, in the present study, we used five molecular markers (four nuclear genes and one mitochondrial gene) to detect hybrids in the trade of pimelodid juvenile fish from different stocks purchased of five seed producers in Brazil. Samples commercialized as pintado (pure species Pseudoplatystoma corruscans) from three fish farms were genetically identified as hybrid cachapinta ( + P. reticulatum $\mathrm{x} \hat{\sigma}^{\lambda}$ P. corruscans). In the stocks purchased as cachandiá (hybrid between + P. reticulatum $\mathrm{x} \delta$ Leiarius marmoratus) and cachapira (hybrid between $q$ P. reticulatum $\mathrm{x} \hat{\delta}$ Phractocephalus hemioliopterus), we suggested the occurrence of intergenus crosses involving the hybrid cachapinta, which was used instead of the pure species P. reticulatum. The problems involving the hybrid cachapinta production were discussed in the present study, especially because these animals have caused genetic contamination and threatened the genetic integrity of natural and cultivated populations. In order to improve the surveillance of the production and provide criteria for the correct management of catfish hybrids, genetic markers has become an excellent alternative to the morphological identification, including juveniles or post-F1 generations.
\end{abstract}

O monitoramento da produção e comércio de híbridos interespecíficos é essencial para o manejo adequado desses animais em pisciculturas. A identificação de híbridos de bagres por análise morfológica não é confiável, especialmente de juvenis e indivíduos pós-F1. Portanto, no presente estudo, cinco marcadores moleculares (quatro genes nucleares e um gene mitocondrial) foram utilizados para detectar híbridos no comércio de juvenis pimelodídeos de diferentes estoques, comprados de cinco produtores de alevinos no Brasil. As amostras comercializadas como pintado (espécie pura Pseudoplatystoma corruscans) foram geneticamente identificadas como híbrido cachapinta ( + P. reticulatum $\mathrm{x} \circlearrowright$ O P. corruscans). Nos estoques comprados como cachandiá (híbrido entre + P. reticulatum x $\widehat{\jmath}$ Leiarius marmoratus) e cachapira (híbrido entre $q P$. reticulatum $\mathrm{x} \widehat{\overbrace{}}$ Phractocephalus hemioliopterus), sugere-se a ocorrência de cruzamentos intergêneros envolvendo o híbrido cachapinta, que foi usado ao invés da espécie pura $P$. reticulatum. Os problemas envolvendo a produção de cachapinta foram discutidos no presente estudo, especialmente porque estes animais têm causado contaminação genética e ameaçado a integridade genética das populações naturais e cultivadas. Com o intuito de melhorar a fiscalização da produção e fornecer critérios para o manejo correto dos híbridos de bagre, marcadores genéticos têm se tornado uma excelente alternativa para a identificação morfológica, incluindo juvenis ou gerações pós-F1.

Keywords: Fish farm, Hybridization, Molecular markers, Nuclear genes, Pimelodidae.

\section{Introduction}

The artificial production of interspecific hybrids consists in a classical method of genetic improvement for aquaculture programs. This technique is widely used for several catfish species of commercial importance, especially in Brazil where about 20 native catfish have been cultivated in aquaculture (Bartley et al., 2001; Hashimoto et al., 2012; Moro et al., 2013). The Neotropical catfish species most often used in aquaculture in South America correspond to the family Pimelodidae, but no data are available about the current situation of pimelodids production in the aquaculture industry (Hashimoto et al., 2015).

${ }^{1}$ Centro de Aquicultura, Universidade Estadual Paulista, UNESP, 14884-900 Jaboticabal, SP, Brazil. diogo@caunesp.unesp.br (corresponding author)

${ }^{2}$ Departamento de Ciências Biológicas, Faculdade de Ciências, Universidade Estadual Paulista, UNESP, 17033-360 Bauru, SP, Brazil. (FDP) ferprado_bio@hotmail.com,(FPF) fpforesti@fc.unesp.br

${ }^{3}$ Departamento de Morfologia, Instituto de Biociências, Universidade Estadual Paulista, UNESP, 18618-970 Botucatu, SP, Brazil. fforesti@ibb.unesp.br 
Nowadays, through interspecific hybridization, catfish species have been highlighted in the aquaculture production of South America fish farms. The hybrids between Pseudoplatystoma reticulatum Eingenmann \& Eigenmann, 1889 (cachara) and Pseudoplatystoma corruscans (Spix \& Agassiz 1829) (pintado) have represented the most economically important catfish hybrid during the last years in Brazil. However, hybridization programs have currently involved fish from other genera, such as Leiarius marmoratus (Gill 1870) (jundiá amazônico) and Phractocephalus hemioliopterus (Bloch \& Schneider 1801) (pirarara), aiming to produce differentiated products and solve problems associated with feeding and cannibalism that can be observed in the early stages of development of these species (Campos, 2010; Porto-Foresti et al., 2010,2013). Moreover, $P$. reticulatum has a longer reproductive period and it is the species most used by the fish farmers as the female parental to generate the interspecific hybrids, named cachapinta (crossed with $P$. corruscans), cachandiá (crossed with L. marmoratus) and cachapira (crossed with P. hemioliopterus) (Campos, 2010; Porto-Foresti et al., 2010, 2013).

The production of catfish hybrids is still incipient in Brazil, particularly due to lack of biological studies, such as reproduction, larviculture, health, nutrition and genetics (Hashimoto et al., 2015). Catfish hybrids are not listed in the official fisheries statistics (IBAMA, 2007), which can be justified by the lack of correct nomenclature or methodologies for the identification of these fish.

The core problem involving fish hybrids is the difficulty of morphological identification, particularly of juvenile individuals and post-F1 hybrids (Allendorf et al., 2001; Hashimoto et al., 2011, 2014b), resulting in several negative impacts. In fish farms, a mistaken trade of fish can occurs (e.g., Serrasalmid hybrids), which is not productive for fish farming, especially when juvenile hybrids can be traded as pure species (Hashimoto et al., 2011, 2014b). Furthermore, hybrids can be used as broodstock resulting in high mortality rates or lost of the hybrid vigor in the offspring (Mia et al., 2005; Hashimoto et al., 2013). In the environment, if released or escape to the rivers, hybrids can backcross with the native pure species, causing genetic introgression and contaminating the genetic pool of pure stocks (Prado et al., 2012a, 2012b; Vaini et al., 2014).

Lately, an increasing concern about the scenario of hybridization has been verified in Brazil, resulting in the necessity of studies to elucidate the commercial and environmental impacts that these animals might represent for pure stocks (Hashimoto et al., 2012). Thus, this study aimed to evaluate the trade of juvenile catfish between seed suppliers and fish farmers in Brazil using molecular markers (four nuclear loci and 1 mitochondrial gene), previously described in others studies (Prado et al., 2011; Hashimoto et al., 2013; Porto-Foresti et al., 2013), particularly for the genetic identification of F1 and post-F1 catfish hybrids.

\section{Material and Methods}

Sample collection. The analyses of molecular identification was carried out in 168 juvenile individuals from five stocks of live fish purchased from several private Brazilian aquaculture seed producers, hereinafter referred to as fish farm A, B, C, D and E (Table 1). All fish farms assessed in this study represent large companies in Brazil. From fish farm A (located in the Mato Grosso do Sul State), B and C (both located in the São Paulo State), we analyzed stocks that were commercially available and labeled as pure species of pintado ( $P$. corruscans). From fish farm $\mathrm{D}$, which is located in the Goiás State, we analyzed one commercially produced stock labeled as hybrid cachandiá ( P P. reticulatum $\mathrm{x} \hat{O}^{\lambda}$ L. marmoratus). From fish farm E, which is located in the São Paulo State, we analyzed one stock that was commercially labeled as hybrid cachapira ( $($ P P. reticulatum $\mathrm{x}$ o P. hemioliopterus). The size of the analyzed fish ranged from 8 to $10 \mathrm{~cm}$. We did not notify the producers that the fish would be used for identification purposes. The pure parental species (samples used as control for reaction specificity of the present study) correspond to the animals analyzed in Porto-Foresti et al. (2013). This study was conducted in strict accordance with the recommendations of the National Council for Control of Animal Experimentation (Brazilian Ministry for Science, Technology and Innovation). The present study was performed under authorization $\mathrm{N}^{\circ} 33435-1$ issued through ICMBio (Chico Mendes Institute for the Conservation of Biodiversity, Brazilian Ministry for Environment). The juvenile fish were not sacrificed for sample collection, i.e., all fish were kept alive in fish farm for further studies. Traditional taxonomic tools for species identification were not used because we analyzed juvenile fish (morphological characters were not yet defined as in adult samples).

Table 1. Molecular identification of juvenile catfish fish stocks purchased from different fish farmers producers.

\begin{tabular}{lcc}
\hline Fish farm & Stocks purchased & $\mathbf{n}$ \\
\hline A (São Paulo State) & Pintado - Pseudoplatystoma corruscans & 20 \\
B (São Paulo State) & Pintado - P. corruscans & 20 \\
C (Mato Grosso do Sul State) & Pintado - P. corruscans & 50 \\
D (Goiás State) & Hybrid cachandiá (Pseudoplatystoma reticulatum x Leiarius marmoratus) & 41 \\
E (São Paulo State) & Hybrid cachapira (P. reticulatum x Phractocephalus hemioliopterus $)$ & 37 \\
\hline
\end{tabular}


DNA extraction. From fin fragments was performed using the Wizard Genomic DNA Purification Kit (Promega) according to the manufacturer's protocol. DNA quantity was assessed against a molecular marker standard (the Low DNA Mass Ladder from Invitrogen) by electrophoresis on a $1 \%$ agarose gel.

Molecular markers. The genetic identification of the stocks was carried out through the PCR-RFLP technique of the nuclear gene ribosomal RNA $18 S$ (18S); and the multiplexPCR method using the nuclear genes recombination activating gene 2 (rag2), elongation factor 1-alpha (ef1 $\alpha$ ), $\beta$-globin (glob), and the mitochondrial gene 16S (16S) (Prado et al., 2011; Hashimoto et al., 2013; Porto-Foresti et al., 2013). Thus, we used four nuclear loci and one mitochondrial gene as potential diagnostic markers for differentiating between the studied species.

In the PCR-RFLP technique, after amplification of the $18 \mathrm{~S}$ gene, enzymatic restriction was performed in a total reaction of $8 \mu \mathrm{L}$ containing: $4 \mu \mathrm{L}$ of the PCR products, $1 \mathrm{X}$ enzyme buffer, 5 units (U) of the restriction enzyme SmaI
$(10 \mathrm{U} / \mu \mathrm{L})(\mathrm{New}$ England Biolabs Inc). The reactions were incubated at $25^{\circ} \mathrm{C}$ for $1 \mathrm{~h}$.

In the multiplex-PCR, we used both universal and species-specific primers in a single reaction for each gene, which generated diagnostic fragments for the studied species (Table 2). Specifically for the genes rag2 and 16S, according to the fish samples of the stocks that were analyzed, we used different combinations of specific primers to facilitate the Multiplex reactions (Table 2), following protocols of PortoForesti et al. (2013). Amplifications were performed by PCR in a thermocycler (Mastercycler personal, Eppendorf) under the conditions (PCR reagents and cycles) described in previous studies (Prado et al., 2011; Hashimoto et al., 2013; Porto-Foresti et al., 2013).

DNA samples from the pure parental species were used as controls for reaction specificity in all experiments. PCR fragment sizes were visualized by electrophoresis on $1.5 \%$ agarose gels stained with ethidium bromide $(1 \mathrm{ng} / \mathrm{ml})$ and visualized under UV illumination. Images were captured using a digital camera (Olympus CAMEDIA C-5060, 5.1 Megapixel, Brazil).

Table 2. Sequences of the primers used in the PCR experiments. Underlined, species-specific primers used for multiplexPCR; Bold, universal primers; F: foward, R: reverse, Pc: Pseudoplatystoma corruscans, Pr: Pseudoplatystoma reticulatum, Lm: Leiarius marmoratus, Ph: Phractocephalus hemioliopterus. ${ }^{a}$ Palumbi, 1996; ${ }^{b}$ Prado et al., 2011; ' Porto-Foresti et al., 2013; ' Moyer et al., 2004; ${ }^{\mathrm{e}}$ Hashimoto et al., 2013; ${ }^{\mathrm{f}}$ White et al., 1990. Combinations of primers for multiplex-PCR of the 16S gene: stocks A, B and C (16S F, 16S R, 16S PcF and 16S PrR); D (16S F, 16S R, 16S PcF, 16S PrR and 16S LmR); and E (16S F, 16S R, 16S PcF, 16S PrR and 16S PhF). Combinations of primers for multiplex-PCR of the RAG2 gene: stocks A, B and C (RAG2 SiluF, RAG2 SiluR, RAG2 PcR and RAG2 PrR); D (RAG2 SiluF, RAG2 SiluR, RAG2 PcR, RAG2 PrR and RAG2 LmR); and E (RAG2 SiluF, RAG2 SiluR, RAG2 PcR, RAG2 PrR and RAG2 PhF).

\begin{tabular}{|c|c|c|c|c|c|c|}
\hline \multirow{2}{*}{ Gene } & \multicolumn{4}{|c|}{ Diagnostic fragment size (bp) } & \multirow{2}{*}{ Primers } & \multirow{2}{*}{ Primer sequences ( $5^{\prime}$ to $\left.3^{\prime}\right)$} \\
\hline & $\mathrm{Pc}$ & $\operatorname{Pr}$ & $\mathrm{Lm}$ & $\mathrm{Ph}$ & & \\
\hline \multirow{6}{*}{$16 \mathrm{~S}$} & \multirow{6}{*}{197} & \multirow{6}{*}{388} & \multirow{6}{*}{307} & \multirow{6}{*}{320} & ${ }^{\mathrm{a}} 16 \mathrm{~S} \mathrm{~F}$ & ACGCCTGTTTATCAAAAACAT \\
\hline & & & & & ${ }^{\mathrm{a}} 16 \mathrm{~S} \mathrm{R}$ & CCGGTCTGAACTCAGATCACGT \\
\hline & & & & & b $\underline{16 \mathrm{~S} \mathrm{PcF}}$ & TGACCATAAAGATCCGGCTAT \\
\hline & & & & & ${ }^{\mathrm{b}} \underline{16 \mathrm{~S} \operatorname{PrR}}$ & TCTTGGTTTTGGGGTTGTTA \\
\hline & & & & & c $\underline{16 \mathrm{~S} \mathrm{LmR}}$ & GGTTGCTATTTAGTTTGACTTAGTTC \\
\hline & & & & & ${ }^{\mathrm{c}} 16 \mathrm{~S} \mathrm{PhF}$ & AAACTAAATAGCCACCTGATCCA \\
\hline \multirow{6}{*}{ RAG2 } & \multirow{6}{*}{308} & \multirow{6}{*}{277} & \multirow{6}{*}{170} & \multirow{6}{*}{206} & ${ }^{\mathrm{b}}$ RAG2 SiluF & CCTGAGTGCTACCTTATTCATGGA \\
\hline & & & & & ${ }^{\mathrm{b}}$ RAG2 SiluR & CTTGGGAGGAAGAGACCATC \\
\hline & & & & & ${ }^{\mathrm{b}} \underline{\mathrm{RAG} 2 \mathrm{PcR}}$ & AACTCCAGGTCAATGAGATAAATG \\
\hline & & & & & ${ }^{\mathrm{c}} \underline{\mathrm{RAG} 2 \mathrm{PrR}}$ & GACAGTCCACCATGCAGTTCCAGG \\
\hline & & & & & ${ }^{\mathrm{c}} \underline{\mathrm{RAG} 2 \mathrm{LmR}}$ & CTAAGGGTGTGACCATACCGA \\
\hline & & & & & ${ }^{\mathrm{c}} \mathrm{RAG} 2 \mathrm{PhF}$ & CTGTTCAGCACACACCCTG \\
\hline \multirow{4}{*}{$\mathrm{EF} 1 \alpha$} & \multirow{4}{*}{520} & \multirow{4}{*}{630} & \multirow{4}{*}{-} & \multirow{4}{*}{ - } & ${ }^{\mathrm{d}} \mathbf{E F 1 \alpha} \mathbf{F}$ & ATTGGAACTGTACCTGTGG \\
\hline & & & & & ${ }^{\mathrm{d}} \mathbf{E F 1 \alpha} \mathbf{R}$ & CAGCCTTCTGTGCAGACTT \\
\hline & & & & & ${ }^{\mathrm{e}} \underline{\mathrm{EF} 1 \alpha \mathrm{PcR}}$ & CAACAATGGCAGCATCTCCT \\
\hline & & & & & ${ }^{\mathrm{e}} \underline{\mathrm{EF} 1 \alpha \operatorname{PrR}}$ & ATAAAGGACAAGGACAAGATCG \\
\hline \multirow{4}{*}{ GLOB } & \multirow{4}{*}{304} & \multirow{4}{*}{137} & \multirow{4}{*}{-} & \multirow{4}{*}{-} & ${ }^{\mathrm{e}}$ GLOB SiluF & TCAATATGGTTCACTGGACAGA \\
\hline & & & & & ${ }^{\mathrm{e}}$ GLOB SiluR & CCAAGAAGCTGAAAGTAGACAGT \\
\hline & & & & & ${ }^{\mathrm{e}} \underline{\mathrm{GLOB}} \mathrm{PcR}$ & CAGCCACCTTGGGGTTTCCT \\
\hline & & & & & ${ }^{\mathrm{e}} \underline{\mathrm{GLOB}} \operatorname{PrF}$ & GGTACGTCTAATCTCAGTAATTGAAA \\
\hline \multirow{2}{*}{$18 \mathrm{~S}$} & \multirow{2}{*}{$163+187$} & \multirow{2}{*}{350} & \multirow{2}{*}{-} & \multirow{2}{*}{ - } & ' $18 S$ SiluR & CCATCGAAAAGTTGATAGGG \\
\hline & & & & & ${ }^{\mathrm{f}} 18 \mathrm{~S}$ NS1 & GTAGTCATATGCTTGTCTC \\
\hline
\end{tabular}


Identification of species and hybrids. Individuals that scored as the same species (homozygous) at all five markers were considered pure species ( $P$. corruscans, $P$. reticulatum, L. marmoratus or P. hemioliopterus) (Table 2). Fish samples with genotypes of heterozygous markers at all four nuclear genes, regardless of the mitochondrial gene, were classified as F1 hybrids. Backcrossed or post-F1 individuals were those that scored differently among any of the five markers.

\section{Results}

Molecular identification of the samples purchased as pure $\boldsymbol{P}$. corruscans. In the samples purchased as pure species $P$. corruscans (stocks from the fish farms A, B and $\mathrm{C}$ ), we obtained heterozygous genotypes (i.e., PCR diagnostic fragments of both species $P$. reticulatum and $P$. corruscans, according to the Table 2) at all four nuclear markers: $\operatorname{rag} 2^{\mathrm{Pr}} / \mathrm{rag} 2^{\mathrm{Pc}}$, glob ${ }^{\mathrm{Pr}} / \mathrm{glob}^{\mathrm{Pc}}$, ef $1 \alpha^{\mathrm{Pr}} / \mathrm{ef} 1 \alpha^{\mathrm{Pc}}$, $18 \mathrm{~S}^{\mathrm{Pr}} / 18 \mathrm{~S}^{\mathrm{Pc}}$ (Pr: P. reticulatum, Pc: P. corruscans). So, these results indicated that they actually correspond to F1 hybrids. Moreover, through multiplex-PCR of the mitochondrial marker, these hybrids showed the genotype of the maternal species $P$. reticulatum, which allowed the identification

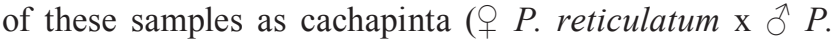
corruscans) instead the reciprocal hybrid pintachara $(q$ $P$. corruscans $\mathrm{x} \delta P$. reticulatum). Thus, the samples of $P$. corruscans were mislabeled because they were actually F1 hybrids cachapinta.

Molecular identification of the samples purchased as hybrid cachandiá. Through multiplex-PCR of the rag2 gene, we observed two distinct electrophoresis patterns in the individuals purchased as hybrid cachandiá: 19 samples were identified with a heterozygous genotype scored for hybrids between $P$. reticulatum and L. marmoratus; however, 22 samples also revealed a heterozygous pattern, but characterized for hybrids between $P$. corruscans and $L$. marmoratus (Table 3). In the analysis of the mitochondrial gene $16 \mathrm{~S}$, all the samples of this stock showed the genotype specific of $P$. reticulatum, even in the individuals scored as hybrids between $P$. corruscans and L. marmoratus, which makes doubtful about the crossing that originated these samples. Thus, these data allowed suggesting the hypothesis of an intergenus crossing between $q$ cachapinta

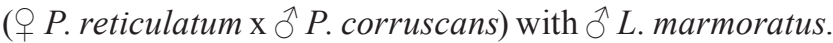

This hypothesis was confirmed through the analyses of additional nuclear markers (glob, efl $\alpha$ and 18S), which showed the occurrence of distinct genotypes in the same individual (Table 3). Therefore, in one single individual, it was possible to detect genotypes of $P$. reticulatum for some genes as well as genotypes of $P$. corruscans for others genes.

Molecular identification of the samples purchased as hybrid cachapira. A similar situation of the cachandiá stock was found for the samples traded as hybrid cachapira, i.e., the analyses of the nuclear gene rag2 demonstrated two types of genotypes: 21 fish were revealed with a heterozygous pattern scored for hybrids between $P$. reticulatum and $P$. hemioliopterus; on the other hand, 16 individuals also showed a heterozygous genotype, but characterized for hybrids between $P$. corruscans and $P$. hemioliopterus (Table 4). In relation to the mitochondrial $16 \mathrm{~S}$, all samples were detected with the diagnostic marker of $P$. hemioliopterus.

Through multiplex-PCR using other nuclear genes (glob, efl $\alpha$ and 18S), we detected the presence of distinct markers in the same individual (Table 4), i.e., genotypes of $P$. reticulatum or $P$. corruscans, depending on the analyzed gene, in a single individual. Consequently, we suggest that the individuals purchased as cachapira correspond to intergenus crossed hybrids between $q P$. hemioliopterus

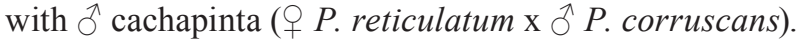

\section{Discussion}

In the last years, several genes were characterized for molecular identification of Neotropical catfish hybrids that are cultivated in Brazilian fish farms (Prado et al., 2011; Hashimoto et al., 2013; Porto-Foresti et al., 2013). In the present study, we demonstrated the effectiveness of these genetic approaches for trade monitoring of juvenile between seed suppliers and fish farmers. Aquaculture industry in Brazil is divided into two complementary sectors: seed trade and adult market (Suplicy, 2007). The seed trade provides juveniles for farmers who perform growout and stocking of the fish for markets. So, the link between seed suppliers and fish farmers is a critical point in the production chain because juvenile stages are morphologically indistinguishable and, consequently, pure species and hybrid stocks may be easily mixed in breeding facilities (Hashimoto et al., 2012). Therefore, our molecular data showed that juvenile catfish hybrids are being wrongly commercialized by fish farms in Brazil, inclusive with the occurrence of intergenus crossings.

In the stocks commercialized as pintado $P$. corruscans, purchased from fish farms A, B and C, we detected through molecular markers that $100 \%$ of the juveniles correspond to hybrid cachapinta, between female $P$. reticulatum and male $P$. corruscans. Possibly, there is a preference on the part of seed suppliers in the production of this hybrid instead of pure species, which can be explained by possible productivity advantages (Crepaldi et al., 2006), or by hatchery problems and difficulties of induced reproduction. It is easier to get spawns of cachara females ( $P$. reticulatum) over a longer period of the year (Campos, 2010); however, pintado ( $P$. corruscans) is more popular in the consumer market, which justifies seed suppliers commercialize mislabeled hybrids as an alternative of pintado. This practice, performed without monitoring, explains the poor statistics on the production and marketing of these animals. Anyway, interspecific hybrids may have desired positive heterosis or hybrid vigor, 
which improves performance due to better growth rates, environmental tolerance and disease resistance (Bartley et al., 2001), although there are not depth studies proving the real advantages of hybrids cachapinta.

The trade of mislabeled juvenile hybrids can generate serious negative consequences for the aquaculture industry and environment. Fish farmers cultivating mislabeled hybrids may perform the improper management for these animals, without the required environmental precautions, resulting in fish hybrids escapes into rivers. The main negative impacts of such unintentional introductions arise because hybrids cachapinta are fertile, allowing the occurrence of backcrosses with their wild pure parental species (Prado et al., 2012a, 2012b). In long term, backcross can cause genetic introgression and contamination of wild pure stocks, and may even lead to local extinction of populations, as observed in several other species (Allendorf et al., 2001; Epifanio \& Philipp, 2001).

Table 3. Individuals of the stock cachandiá and their respective phenotypes of molecular identification using the mitochondrial and nuclear genes. Pc: Pseudoplatystoma corruscans, Pr: P. reticulatum, Lm: Leiarius marmoratus.

\begin{tabular}{|c|c|c|c|c|c|c|c|}
\hline \multirow{2}{*}{ Sample ID } & \multirow{2}{*}{ Fish farm label } & \multirow{2}{*}{$\begin{array}{c}\text { DNAmt } \\
\text { phenotype }\end{array}$} & \multicolumn{4}{|c|}{ Nuclear phenotypes } & \multirow{2}{*}{ Molecular identification } \\
\hline & & & RAG2 & GLOB & $\mathrm{EF} 1 \alpha$ & $18 \mathrm{~S}$ & \\
\hline D01 & cachandiá & $16 \mathrm{~S}^{\operatorname{Pr}}$ & $\operatorname{rag} 2^{\text {Pr }} / \mathrm{rag} 2^{\mathrm{Lm}}$ & glob $^{\mathrm{Pc}}$ & efl $1 \alpha^{\mathrm{Pr}}$ & $18 \mathrm{~S}^{\mathrm{Pc}}$ & intergenus crossing \\
\hline D02 & cachandiá & $16 \mathrm{~S}^{\operatorname{Pr}}$ & $\operatorname{rag} 2^{\mathrm{Pc}} / \mathrm{rag} 2^{\mathrm{Lm}}$ & $\mathrm{glob}^{\mathrm{Pr}}$ & $\mathrm{ef} 1 \alpha^{\mathrm{Pc}}$ & $18 \mathrm{~S}^{\mathrm{Pr}}$ & intergenus crossing \\
\hline D03 & cachandiá & $16 \mathrm{~S}^{\operatorname{Pr}}$ & $\operatorname{rag} 2^{\operatorname{Pr}} / \operatorname{rag} 2^{\mathrm{Lm}}$ & glob $^{\mathrm{Pc}}$ & $\mathrm{ef} 1 \alpha^{\mathrm{Pr}}$ & $18 \mathrm{~S}^{\mathrm{Pc}}$ & intergenus crossing \\
\hline D04 & cachandiá & $16 \mathrm{~S}^{\mathrm{Pr}}$ & $\operatorname{rag} 2^{\mathrm{Pr}} / \mathrm{rag} 2^{\mathrm{Lm}}$ & glob $^{\mathrm{Pc}}$ & ef $1 \alpha^{\mathrm{Pr}}$ & $18 \mathrm{~S}^{\operatorname{Pr}}$ & intergenus crossing \\
\hline D05 & cachandiá & $16 \mathrm{~S}^{\mathrm{Pr}}$ & $\operatorname{rag} 2^{\operatorname{Pr}} / \operatorname{rag} 2^{\mathrm{Lm}}$ & $\mathrm{glob}^{\mathrm{Pc}}$ & ef $1 \alpha^{\mathrm{Pr}}$ & $18 \mathrm{~S}^{\operatorname{Pr}}$ & intergenus crossing \\
\hline D06 & cachandiá & $16 \mathrm{~S}^{\mathrm{Pr}}$ & $\operatorname{rag} 2^{\mathrm{Pc}} / \mathrm{rag} 2^{\mathrm{Lm}}$ & $\operatorname{glob}^{\mathrm{Pr}}$ & ef $1 \alpha^{\mathrm{Pr}}$ & $18 \mathrm{~S}^{\mathrm{Pc}}$ & intergenus crossing \\
\hline D07 & cachandiá & $16 \mathrm{~S}^{\operatorname{Pr}}$ & $\operatorname{rag} 2^{\mathrm{Pc}} / \mathrm{rag} 2^{\mathrm{Lm}}$ & $\mathrm{glob}^{\mathrm{Pr}}$ & $\mathrm{ef} 1 \alpha^{\mathrm{Pc}}$ & $18 \mathrm{~S}^{\mathrm{Pc}}$ & intergenus crossing \\
\hline D08 & cachandiá & $16 \mathrm{~S}^{\operatorname{Pr}}$ & $\operatorname{rag} 2^{\mathrm{Pc}} / \mathrm{rag} 2^{\mathrm{Lm}}$ & $\mathrm{glob}^{\mathrm{Pr}}$ & $\mathrm{ef} 1 \alpha^{\mathrm{Pc}}$ & $18 \mathrm{~S}^{\operatorname{Pr}}$ & intergenus crossing \\
\hline D09 & cachandiá & $16 \mathrm{~S}^{\operatorname{Pr}}$ & $\operatorname{rag} 2^{\mathrm{Pc}} / \mathrm{rag} 2^{\mathrm{Lm}}$ & $\mathrm{glob}^{\mathrm{Pr}}$ & $\mathrm{ef} 1 \alpha^{\mathrm{Pc}}$ & $18 \mathrm{~S}^{\operatorname{Pr}}$ & intergenus crossing \\
\hline D10 & cachandiá & $16 \mathrm{~S}^{\operatorname{Pr}}$ & $\operatorname{rag} 2^{\mathrm{Pc}} / \mathrm{rag} 2^{\mathrm{Lm}}$ & glob $^{\mathrm{Pc}}$ & $\mathrm{ef} 1 \alpha^{\mathrm{Pr}}$ & $18 \mathrm{~S}^{\mathrm{Pc}}$ & intergenus crossing \\
\hline D11 & cachandiá & $16 \mathrm{~S}^{\mathrm{Pr}}$ & $\operatorname{rag} 2^{\mathrm{Pr}} / \mathrm{rag} 2^{\mathrm{Lm}}$ & glob $^{\mathrm{Pc}}$ & $\mathrm{ef} 1 \alpha^{\mathrm{Pr}}$ & $18 \mathrm{~S}^{\mathrm{Pc}}$ & intergenus crossing \\
\hline D12 & cachandiá & $16 \mathrm{~S}^{\mathrm{Pr}}$ & $\operatorname{rag} 2^{\mathrm{Pr}} / \mathrm{rag} 2^{\mathrm{Lm}}$ & glob $^{\mathrm{Pc}}$ & $\mathrm{ef} 1 \alpha^{\mathrm{Pc}}$ & $18 \mathrm{~S}^{\operatorname{Pr}}$ & intergenus crossing \\
\hline D13 & cachandiá & $16 \mathrm{~S}^{\operatorname{Pr}}$ & $\operatorname{rag} 2^{\mathrm{Pc}} / \mathrm{rag} 2^{\mathrm{Lm}}$ & $\mathrm{glob}^{\mathrm{Pr}}$ & $\mathrm{ef} 1 \alpha^{\mathrm{Pr}}$ & $18 \mathrm{~S}^{\operatorname{Pr}}$ & intergenus crossing \\
\hline D14 & cachandiá & $16 \mathrm{~S}^{\operatorname{Pr}}$ & $\operatorname{rag} 2^{\mathrm{Pc}} / \mathrm{rag} 2^{\mathrm{Lm}}$ & glob $^{\mathrm{Pc}}$ & $\mathrm{ef} 1 \alpha^{\mathrm{Pc}}$ & $18 \mathrm{~S}^{\operatorname{Pr}}$ & intergenus crossing \\
\hline D15 & cachandiá & $16 \mathrm{~S}^{\mathrm{Pr}}$ & $\operatorname{rag} 2^{\mathrm{Pc}} / \mathrm{rag} 2^{\mathrm{Lm}}$ & glob $^{\mathrm{Pr}}$ & $\mathrm{ef} 1 \alpha^{\mathrm{Pc}}$ & $18 \mathrm{~S}^{\operatorname{Pr}}$ & intergenus crossing \\
\hline D16 & cachandiá & $16 \mathrm{~S}^{\mathrm{Pr}}$ & $\operatorname{rag} 2^{\operatorname{Pr}} / \operatorname{rag} 2^{\mathrm{Lm}}$ & glob $^{\mathrm{Pc}}$ & ef $1 \alpha^{\mathrm{Pr}}$ & $18 \mathrm{~S}^{\mathrm{Pc}}$ & intergenus crossing \\
\hline D17 & cachandiá & $16 \mathrm{~S}^{\operatorname{Pr}}$ & $\operatorname{rag} 2^{\operatorname{Pr}} / \mathrm{rag} 2^{\mathrm{Lm}}$ & $\mathrm{glob}^{\mathrm{Pc}}$ & $\mathrm{ef} 1 \alpha^{\mathrm{Pc}}$ & $18 \mathrm{~S}^{\operatorname{Pr}}$ & intergenus crossing \\
\hline D18 & cachandiá & $16 \mathrm{~S}^{\operatorname{Pr}}$ & $\mathrm{rag} 2^{\mathrm{Pr}} / \mathrm{rag} 2^{\mathrm{Lm}}$ & $\operatorname{glob}^{\mathrm{Pr}}$ & ef $1 \alpha^{\mathrm{Pc}}$ & $18 \mathrm{~S}^{\operatorname{Pr}}$ & intergenus crossing \\
\hline D19 & cachandiá & $16 \mathrm{~S}^{\mathrm{Pr}}$ & $\operatorname{rag} 2^{\mathrm{Pc}} / \mathrm{rag} 2^{\mathrm{Lm}}$ & glob $^{\mathrm{Pc}}$ & $\mathrm{ef} 1 \alpha^{\mathrm{Pc}}$ & $18 \mathrm{~S}^{\operatorname{Pr}}$ & intergenus crossing \\
\hline D20 & cachandiá & $16 \mathrm{~S}^{\operatorname{Pr}}$ & $\operatorname{rag} 2^{\mathrm{Pc}} / \mathrm{rag} 2^{\mathrm{Lm}}$ & $\mathrm{glob}^{\mathrm{Pr}}$ & $\mathrm{ef} 1 \alpha^{\mathrm{Pc}}$ & $18 \mathrm{~S}^{\mathrm{Pc}}$ & intergenus crossing \\
\hline D21 & cachandiá & $16 S^{\operatorname{Pr}}$ & $\operatorname{rag} 2^{\mathrm{Pr}} / \mathrm{rag} 2^{\mathrm{Lm}}$ & $\mathrm{glob}^{\mathrm{Pr}}$ & $\mathrm{ef} 1 \alpha^{\mathrm{Pc}}$ & $18 \mathrm{~S}^{\mathrm{Pc}}$ & intergenus crossing \\
\hline D22 & cachandiá & $16 \mathrm{~S}^{\operatorname{Pr}}$ & $\operatorname{rag} 2^{\mathrm{Pc}} / \mathrm{rag} 2^{\mathrm{Lm}}$ & $\mathrm{glob}^{\mathrm{Pr}}$ & $\mathrm{ef} 1 \alpha^{\mathrm{Pr}}$ & $18 \mathrm{~S}^{\mathrm{Pc}}$ & intergenus crossing \\
\hline D23 & cachandiá & $16 \mathrm{~S}^{\mathrm{Pr}}$ & $\operatorname{rag} 2^{\mathrm{Pc}} / \mathrm{rag} 2^{\mathrm{Lm}}$ & glob $^{\mathrm{Pc}}$ & $\mathrm{ef} 1 \alpha^{\mathrm{Pc}}$ & $18 \mathrm{~S}^{\operatorname{Pr}}$ & intergenus crossing \\
\hline D24 & cachandiá & $16 \mathrm{~S}^{\operatorname{Pr}}$ & $\operatorname{rag} 2^{\mathrm{Pr}} / \mathrm{rag} 2^{\mathrm{Lm}}$ & $\mathrm{glob}^{\mathrm{Pr}}$ & $\mathrm{ef} 1 \alpha^{\mathrm{Pc}}$ & $18 \mathrm{~S}^{\mathrm{Pc}}$ & intergenus crossing \\
\hline D25 & cachandiá & $16 \mathrm{~S}^{\operatorname{Pr}}$ & $\operatorname{rag} 2^{\mathrm{Pc}} / \mathrm{rag} 2^{\mathrm{Lm}}$ & $\mathrm{glob}^{\mathrm{Pr}}$ & $\mathrm{ef} 1 \alpha^{\mathrm{Pc}}$ & $18 \mathrm{~S}^{\operatorname{Pr}}$ & intergenus crossing \\
\hline D26 & cachandiá & $16 \mathrm{~S}^{\operatorname{Pr}}$ & $\operatorname{rag} 2^{\mathrm{Pc}} / \operatorname{rag} 2^{\mathrm{Lm}}$ & $\operatorname{glob}^{\mathrm{Pr}}$ & ef $1 \alpha^{\mathrm{Pr}}$ & $18 \mathrm{~S}^{\mathrm{Pc}}$ & intergenus crossing \\
\hline D27 & cachandiá & $16 \mathrm{~S}^{\mathrm{Pr}}$ & $\operatorname{rag} 2^{\mathrm{Pc}} / \mathrm{rag} 2^{\mathrm{Lm}}$ & glob $^{\mathrm{Pc}}$ & $\mathrm{ef} 1 \alpha^{\mathrm{Pr}}$ & $18 \mathrm{~S}^{\mathrm{Pc}}$ & intergenus crossing \\
\hline D28 & cachandiá & $16 \mathrm{~S}^{\operatorname{Pr}}$ & $\operatorname{rag} 2^{\mathrm{Pr}} / \mathrm{rag} 2^{\mathrm{Lm}}$ & $\mathrm{glob}^{\mathrm{Pr}}$ & $\mathrm{ef} 1 \alpha^{\mathrm{Pc}}$ & $18 \mathrm{~S}^{\operatorname{Pr}}$ & intergenus crossing \\
\hline D29 & cachandiá & $16 \mathrm{~S}^{\operatorname{Pr}}$ & $\operatorname{rag} 2^{\operatorname{Pr}} / \operatorname{rag} 2^{\mathrm{Lm}}$ & $\mathrm{glob}^{\mathrm{Pr}}$ & $\mathrm{ef} 1 \alpha^{\mathrm{Pc}}$ & $18 \mathrm{~S}^{\mathrm{Pc}}$ & intergenus crossing \\
\hline D30 & cachandiá & $16 \mathrm{~S}^{\operatorname{Pr}}$ & $\operatorname{rag} 2^{\mathrm{Pc}} / \mathrm{rag} 2^{\mathrm{Lm}}$ & $\mathrm{glob}^{\mathrm{Pc}}$ & ef $1 \alpha^{\mathrm{Pr}}$ & $18 \mathrm{~S}^{\mathrm{Pc}}$ & intergenus crossing \\
\hline D31 & cachandiá & $16 \mathrm{~S}^{\operatorname{Pr}}$ & $\operatorname{rag} 2^{\operatorname{Pr}} / \mathrm{rag} 2^{\mathrm{Lm}}$ & $\mathrm{glob}^{\mathrm{Pr}}$ & $\mathrm{ef} 1 \alpha^{\mathrm{Pc}}$ & $18 \mathrm{~S}^{\operatorname{Pr}}$ & intergenus crossing \\
\hline D32 & cachandiá & $16 \mathrm{~S}^{\operatorname{Pr}}$ & $\operatorname{rag} 2^{\operatorname{Pr}} / \operatorname{rag} 2^{\mathrm{Lm}}$ & $\mathrm{glob}^{\mathrm{Pr}}$ & $\mathrm{ef} 1 \alpha^{\mathrm{Pc}}$ & $18 \mathrm{~S}^{\operatorname{Pr}}$ & intergenus crossing \\
\hline D33 & cachandiá & $16 \mathrm{~S}^{\operatorname{Pr}}$ & $\mathrm{rag} 2^{\mathrm{Pc}} / \mathrm{rag} 2^{\mathrm{Lm}}$ & $\operatorname{glob}^{\mathrm{Pr}}$ & ef $1 \alpha^{\mathrm{Pr}}$ & $18 \mathrm{~S}^{\operatorname{Pr}}$ & intergenus crossing \\
\hline D34 & cachandiá & $16 \mathrm{~S}^{\operatorname{Pr}}$ & $\operatorname{rag} 2^{\mathrm{Pc}} / \mathrm{rag} 2^{\mathrm{Lm}}$ & $\operatorname{glob}^{\mathrm{Pr}}$ & $\mathrm{ef} 1 \alpha^{\mathrm{Pc}}$ & $18 \mathrm{~S}^{\mathrm{Pc}}$ & intergenus crossing \\
\hline D35 & cachandiá & $16 \mathrm{~S}^{\operatorname{Pr}}$ & $\operatorname{rag} 2^{\mathrm{Pc}} / \mathrm{rag} 2^{\mathrm{Lm}}$ & glob $^{\mathrm{Pc}}$ & $\mathrm{ef} 1 \alpha^{\mathrm{Pc}}$ & $18 \mathrm{~S}^{\operatorname{Pr}}$ & intergenus crossing \\
\hline D36 & cachandiá & $16 \mathrm{~S}^{\mathrm{Pr}}$ & $\operatorname{rag} 2^{\mathrm{Pr}} / \mathrm{rag} 2^{\mathrm{Lm}}$ & $\mathrm{glob}^{\mathrm{Pc}}$ & ef $1 \alpha^{\mathrm{Pr}}$ & $18 \mathrm{~S}^{\mathrm{Pc}}$ & intergenus crossing \\
\hline D37 & cachandiá & $16 \mathrm{~S}^{\operatorname{Pr}}$ & $\operatorname{rag} 2^{\operatorname{Pr}} / \operatorname{rag} 2^{\mathrm{Lm}}$ & glob $^{\mathrm{Pc}}$ & $\mathrm{ef} 1 \alpha^{\mathrm{Pr}}$ & $18 \mathrm{~S}^{\operatorname{Pr}}$ & intergenus crossing \\
\hline D38 & cachandiá & $16 \mathrm{~S}^{\mathrm{Pr}}$ & $\operatorname{rag} 2^{\mathrm{Pc}} / \operatorname{rag} 2^{\mathrm{Lm}}$ & glob $^{\mathrm{Pc}}$ & $\mathrm{ef} 1 \alpha^{\mathrm{Pc}}$ & $18 \mathrm{~S}^{\operatorname{Pr}}$ & intergenus crossing \\
\hline D39 & cachandiá & $16 \mathrm{~S}^{\operatorname{Pr}}$ & $\operatorname{rag} 2^{\mathrm{Pr}} / \mathrm{rag} 2^{\mathrm{Lm}}$ & $\mathrm{glob}^{\mathrm{Pr}}$ & $\mathrm{ef} 1 \alpha^{\mathrm{Pc}}$ & $18 \mathrm{~S}^{\mathrm{Pc}}$ & intergenus crossing \\
\hline D40 & cachandiá & $16 \mathrm{~S}^{\operatorname{Pr}}$ & $\mathrm{rag} 2^{\mathrm{Pr}} / \mathrm{rag} 2^{\mathrm{Lm}}$ & $\mathrm{glob}^{\mathrm{Pc}}$ & ef $1 \alpha^{\mathrm{Pc}}$ & $18 \mathrm{~S}^{\operatorname{Pr}}$ & intergenus crossing \\
\hline D41 & cachandiá & $16 \mathrm{~S}^{\operatorname{Pr}}$ & $\operatorname{rag} 2^{\mathrm{Pc}} / \mathrm{rag} 2^{\mathrm{Lm}}$ & $\mathrm{glob}^{\mathrm{Pc}}$ & ef $1 \alpha^{\mathrm{Pr}}$ & $18 \mathrm{~S}^{\mathrm{Pc}}$ & intergenus crossing \\
\hline
\end{tabular}


Table 4. Individuals of the stock cachapira and their respective genotypes of molecular identification using the mitochondrial and nuclear genes. Pc: Pseudoplatystoma corruscans, Pr: P. reticulatum, Ph: Phractocephalus hemioliopterus.

\begin{tabular}{|c|c|c|c|c|c|c|c|}
\hline \multirow{2}{*}{ Sample ID } & \multirow{2}{*}{ Fish farm label } & \multirow{2}{*}{$\begin{array}{c}\text { DNAmt } \\
\text { phenotype }\end{array}$} & \multicolumn{4}{|c|}{ Nuclear phenotypes } & \multirow{2}{*}{ Molecular identification } \\
\hline & & & RAG2 & GLOB & $\mathrm{EF} 1 \alpha$ & $18 \mathrm{~S}$ & \\
\hline E01 & cachapira & $16 \mathrm{~S}^{\mathrm{Ph}}$ & $\operatorname{rag} 2^{\mathrm{Pc}} / \mathrm{rag} 2^{\mathrm{Ph}}$ & glob $^{\text {Pr }}$ & efl $1 \alpha^{\text {Pr }}$ & $18 \mathrm{~S}^{\mathrm{Pc}}$ & intergenus crossing \\
\hline E02 & cachapira & $16 \mathrm{~S}^{\mathrm{Ph}}$ & $\operatorname{rag} 2^{\mathrm{Pc}} / \mathrm{rag} 2^{\mathrm{Ph}}$ & glob $^{\mathrm{Pr}}$ & efl $\alpha^{\mathrm{Pc}}$ & $18 \mathrm{~S}^{\operatorname{Pr}}$ & intergenus crossing \\
\hline E03 & cachapira & $16 \mathrm{~S}^{\mathrm{Ph}}$ & $\operatorname{rag} 2^{\mathrm{Pc}} / \mathrm{rag} 2^{\mathrm{Ph}}$ & glob $^{\mathrm{Pc}}$ & $\mathrm{ef} 1 \alpha^{\mathrm{Pr}}$ & $18 \mathrm{~S}^{\mathrm{Pc}}$ & intergenus crossing \\
\hline E04 & cachapira & $16 \mathrm{~S}^{\mathrm{Ph}}$ & $\operatorname{rag} 2^{\mathrm{Pr}} / \mathrm{rag} 2^{\mathrm{Ph}}$ & $\operatorname{glob}^{\mathrm{Pc}}$ & ef $1 \alpha^{\mathrm{Pc}}$ & $18 \mathrm{~S}^{\operatorname{Pr}}$ & intergenus crossing \\
\hline E05 & cachapira & $16 \mathrm{~S}^{\mathrm{Ph}}$ & $\operatorname{rag} 2^{\mathrm{Pc}} / \mathrm{rag} 2^{\mathrm{Ph}}$ & $\operatorname{glob}^{\mathrm{Pc}}$ & efl $\alpha^{\mathrm{Pr}}$ & $18 \mathrm{~S}^{\mathrm{Pc}}$ & intergenus crossing \\
\hline E07 & cachapira & $16 \mathrm{~S}^{\mathrm{Ph}}$ & $\operatorname{rag} 2^{\mathrm{Pr}} / \mathrm{rag} 2^{\mathrm{Ph}}$ & glob $^{\mathrm{Pc}}$ & ef $1 \alpha^{\mathrm{Pc}}$ & $18 \mathrm{~S}^{\operatorname{Pr}}$ & intergenus crossing \\
\hline E08 & cachapira & $16 \mathrm{~S}^{\mathrm{Ph}}$ & $\operatorname{rag} 2^{\mathrm{Pr}} / \mathrm{rag} 2^{\mathrm{Ph}}$ & glob $^{\mathrm{Pr}}$ & efl $\alpha^{\mathrm{Pr}}$ & $18 \mathrm{~S}^{\mathrm{Pc}}$ & intergenus crossing \\
\hline E09 & cachapira & $16 \mathrm{~S}^{\mathrm{Ph}}$ & $\operatorname{rag} 2^{\mathrm{Pc}} / \mathrm{rag} 2^{\mathrm{Ph}}$ & glob $^{\mathrm{Pr}}$ & ef $1 \alpha^{\mathrm{Pc}}$ & $18 \mathrm{~S}^{\mathrm{Pc}}$ & intergenus crossing \\
\hline E10 & cachapira & $16 \mathrm{~S}^{\mathrm{Ph}}$ & $\operatorname{rag} 2^{\mathrm{Pr}} / \mathrm{rag} 2^{\mathrm{Ph}}$ & glob $^{\mathrm{Pr}}$ & $\mathrm{ef} 1 \alpha^{\mathrm{Pr}}$ & $18 \mathrm{~S}^{\mathrm{Pc}}$ & intergenus crossing \\
\hline E11 & cachapira & $16 \mathrm{~S}^{\mathrm{Ph}}$ & $\operatorname{rag} 2^{\mathrm{Pc}} / \mathrm{rag} 2^{\mathrm{Ph}}$ & $\operatorname{glob}^{\mathrm{Pr}}$ & efl $\alpha^{\operatorname{Pr}}$ & $18 \mathrm{~S}^{\mathrm{Pc}}$ & intergenus crossing \\
\hline E16 & cachapira & $16 \mathrm{~S}^{\mathrm{Ph}}$ & $\operatorname{rag} 2^{\mathrm{Pr}} / \mathrm{rag} 2^{\mathrm{Ph}}$ & glob $^{\mathrm{Pr}}$ & ef $1 \alpha^{\mathrm{Pc}}$ & $18 \mathrm{~S}^{\mathrm{Pr}}$ & intergenus crossing \\
\hline E17 & cachapira & $16 \mathrm{~S}^{\mathrm{Ph}}$ & $\operatorname{rag} 2^{\mathrm{Pr}} / \mathrm{rag} 2^{\mathrm{Ph}}$ & glob $^{\mathrm{Pc}}$ & $\mathrm{ef} 1 \alpha^{\mathrm{Pr}}$ & $18 \mathrm{~S}^{\mathrm{Pc}}$ & intergenus crossing \\
\hline E18 & cachapira & $16 \mathrm{~S}^{\mathrm{Ph}}$ & $\operatorname{rag} 2^{\mathrm{Pc}} / \mathrm{rag} 2^{\mathrm{Ph}}$ & $\operatorname{glob}^{\mathrm{Pc}}$ & efl $1 \alpha^{\mathrm{Pc}}$ & $18 \mathrm{~S}^{\operatorname{Pr}}$ & intergenus crossing \\
\hline E19 & cachapira & $16 \mathrm{~S}^{\mathrm{Ph}}$ & $\operatorname{rag} 2^{\mathrm{Pr}} / \mathrm{rag} 2^{\mathrm{Ph}}$ & glob $^{\mathrm{Pc}}$ & ef $1 \alpha^{\mathrm{Pc}}$ & $18 \mathrm{~S}^{\mathrm{Pc}}$ & intergenus crossing \\
\hline E20 & cachapira & $16 \mathrm{~S}^{\mathrm{Ph}}$ & $\operatorname{rag} 2^{\mathrm{Pr}} / \mathrm{rag} 2^{\mathrm{Ph}}$ & glob $^{\mathrm{Pr}}$ & efl $\alpha^{\mathrm{Pr}}$ & $18 \mathrm{~S}^{\mathrm{Pc}}$ & intergenus crossing \\
\hline E21 & cachapira & $16 \mathrm{~S}^{\mathrm{Ph}}$ & $\operatorname{rag} 2^{\mathrm{Pr}} / \mathrm{rag} 2^{\mathrm{Ph}}$ & $\mathrm{glob}^{\mathrm{Pc}}$ & ef $1 \alpha^{\mathrm{Pc}}$ & $18 \mathrm{~S}^{\operatorname{Pr}}$ & intergenus crossing \\
\hline E22 & cachapira & $16 \mathrm{~S}^{\mathrm{Ph}}$ & $\operatorname{rag} 2^{\mathrm{Pr}} / \mathrm{rag} 2^{\mathrm{Ph}}$ & $\operatorname{glob}^{\mathrm{Pc}}$ & efl $\alpha^{\mathrm{Pr}}$ & $18 \mathrm{~S}^{\operatorname{Pr}}$ & intergenus crossing \\
\hline E23 & cachapira & $16 \mathrm{~S}^{\mathrm{Ph}}$ & $\operatorname{rag} 2^{\mathrm{Pc}} / \mathrm{rag} 2^{\mathrm{Ph}}$ & $\operatorname{glob}^{\mathrm{Pc}}$ & ef $1 \alpha^{\mathrm{Pc}}$ & $18 \mathrm{~S}^{\operatorname{Pr}}$ & intergenus crossing \\
\hline E24 & cachapira & $16 \mathrm{~S}^{\mathrm{Ph}}$ & $\operatorname{rag} 2^{\mathrm{Pr}} / \mathrm{rag} 2^{\mathrm{Ph}}$ & $\operatorname{glob}^{\mathrm{Pr}}$ & efl $1 \alpha^{\mathrm{Pr}}$ & $18 \mathrm{~S}^{\mathrm{Pc}}$ & intergenus crossing \\
\hline E32 & cachapira & $16 \mathrm{~S}^{\mathrm{Ph}}$ & $\operatorname{rag} 2^{\mathrm{Pc}} / \mathrm{rag} 2^{\mathrm{Ph}}$ & glob $^{\mathrm{Pc}}$ & efl $1 \alpha^{\operatorname{Pr}}$ & $18 \mathrm{~S}^{\mathrm{Pc}}$ & intergenus crossing \\
\hline E33 & cachapira & $16 \mathrm{~S}^{\mathrm{Ph}}$ & $\operatorname{rag} 2^{\mathrm{Pc}} / \mathrm{rag} 2^{\mathrm{Ph}}$ & glob $^{\mathrm{Pr}}$ & $\mathrm{ef} 1 \alpha^{\mathrm{Pr}}$ & $18 \mathrm{~S}^{\mathrm{Pc}}$ & intergenus crossing \\
\hline E34 & cachapira & $16 \mathrm{~S}^{\mathrm{Ph}}$ & $\operatorname{rag} 2^{\mathrm{Pc}} / \mathrm{rag} 2^{\mathrm{Ph}}$ & glob $^{\mathrm{Pc}}$ & $\mathrm{ef} 1 \alpha^{\mathrm{Pr}}$ & $18 \mathrm{~S}^{\operatorname{Pr}}$ & intergenus crossing \\
\hline E35 & cachapira & $16 \mathrm{~S}^{\mathrm{Ph}}$ & $\operatorname{rag} 2^{\mathrm{Pr}} / \mathrm{rag} 2^{\mathrm{Ph}}$ & glob $^{\mathrm{Pr}}$ & ef $1 \alpha^{\mathrm{Pc}}$ & $18 \mathrm{~S}^{\operatorname{Pr}}$ & intergenus crossing \\
\hline E36 & cachapira & $16 \mathrm{~S}^{\mathrm{Ph}}$ & $\operatorname{rag} 2^{\mathrm{Pc}} / \mathrm{rag} 2^{\mathrm{Ph}}$ & glob $^{\mathrm{Pr}}$ & ef $1 \alpha^{\mathrm{Pc}}$ & $18 \mathrm{~S}^{\operatorname{Pr}}$ & intergenus crossing \\
\hline E37 & cachapira & $16 \mathrm{~S}^{\mathrm{Ph}}$ & $\operatorname{rag} 2^{\mathrm{Pc}} / \mathrm{rag} 2^{\mathrm{Ph}}$ & glob $^{\mathrm{Pc}}$ & $\mathrm{ef} 1 \alpha^{\mathrm{Pr}}$ & $18 \mathrm{~S}^{\operatorname{Pr}}$ & intergenus crossing \\
\hline
\end{tabular}

In fact, the occurrence of hybrid cachapinta has already been detected in natural environments in Brazil (Prado et al., 2012b; Vaini et al., 2014), threatening natural populations, possibly due to escapes of aquaculture systems. Therefore, the trade and management of hybrids cachapinta need receive special attention in the aquaculture industry, especially because hybrids are sold commercially as one of the parental species (present study). A similar situation has also been reported to hybrids of the Serrasalmidae, in which mislabeled juveniles of hybrid tambacu (female Colossoma macropomum $\mathrm{x}$ male Piaractus mesopotamicus) are sold as pure species
(Hashimoto et al., 2011). In this case, hybrids tambacu have also been reported in sympatry with pure species in Brazilian rivers (Orsi \& Agostinho, 1999; Paiva et al., 2002).

The establishment of broodstocks composed by hybrids is an additional negative consequence of the trade of mislabeled hybrids cachapinta (Hashimoto et al., 2013; present study). According to Hashimoto et al. (2013), breeding stocks of $P$. corruscans and $P$. reticulatum may suffer contamination by hybrids in fish farms, especially by post-F1 individuals, causing production losses in aquaculture systems due to high juvenile mortality and 
individuals with abnormality. Furthermore, productive advantages associated with hybrid vigor may be absent in post-F1 generations because introgressive hybridization decreases the heterosis obtained in F1 hybrids (Hashimoto et al., 2012).

In addition, our data emphasize another aspect of the problem involving the fertility of hybrids cachapinta, through the molecular identification of stocks marketed as cachandiá and cachapira from fish farms D and FE, respectively. With the use of five molecular markers (4 nuclear loci and one mitochondrial gene), we suggested the occurrence of post-F1 individuals in both stocks of cachandiá and cachapira, in which hybrids cachapinta were used as breeder in crossings with species of other genera (Leiarius marmoratus and Phractocephalus hemioliopterus). In Brazil, a similar situation was also described for hybrids of the Serrasalmidae family (Hashimoto et al., 2014b), in which the interspecific

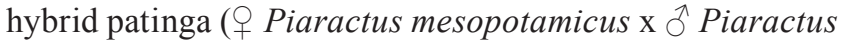
brachypomus) was crossed with a species of different genus (Colossoma macropomum).

According to the results of the present study for the Pimelodidade family, we demonstrated the heterogeneity of interspecific and intergenus crosses that have been performed in Brazilian fish farms, which makes more difficulty the identification of juvenile fish by traditional morphological data. Moreover, fish farmers do not have awareness about the negative impacts resultant from the erroneous use of hybrids. Therefore, the data herein obtained reinforces the importance of genetic monitoring for the appropriate management of these animals and to avoid the problems related with genetic introgression (Hashimoto et al., 2010, 2014a), threatening the genetic integrity of wild pure species and causing production losses in the aquaculture industry.

In conclusion, our data of molecular identification show the necessity of an adequate management of the fish stocks in fish farms and, consequently, a better organization of the Brazilian aquaculture industry.

\section{Acknowledgements}

This work was supported by grants from Fundação de Amparo à Pesquisa do Estado de São Paulo (Proc. FAPESP n. 2014/03772-7) and Conselho Nacional de Desenvolvimento Científico e Tecnológico (CNPq 446779/2014-8). All authors equally contributed in the present study.

\section{References}

Allendorf, F. W., R. F. Leary, P. Spruell \& J. K. Wenburg. 2001. The problems with hybrids: setting conservation guidelines. Trends in Ecology \& Evolution, 16: 613-622.

Bartley, D. M., K. Rana \& A. J. Immink. 2001. The use of interspecific hybrids in aquaculture and fisheries. Reviews in Fish Biology and Fisheries, 10: 325-337.
Campos, J. L. 2010. O cultivo do pintado (Pseudoplatystoma corruscans, Spix; Agassiz, 1829), outras espécies do gênero Pseudoplatystoma e seus híbridos. Pp. 335-361. In: Baldisserotto, B. \& L. C. Gomes (Orgs.). Espécies nativas para a piscicultura no Brasil. Universidade Federal de Santa Maria, Santa Maria, Brazil.

Crepaldi, D. V., P. M. C. Faria, E. A. Teixeira, L. P. Ribeiro, A. A. P. Costa, D. C. Melo, A. P. R. Cintra, S. A. Prado, F. A. A. Costa, M. L. Drumond, V. E. Lopes \&V. E. Moraes. 2006. O surubim na aquacultura do Brasil. Revista Brasileira de Reprodução Animal, 30: 150-158.

Epifanio, J. \& D. Philipp. 2001. Simulating the extinction of parental lineages from introgressive hybridization: the effects of fitness, initial proportions of parental taxa, and mate choice. Reviews in Fish Biology and Fisheries, 10: 339-354.

Hashimoto, D. T., F. F. Mendonça, J. A. Senhorini, J. Bortolozzi, C. Oliveira, F. Foresti \& F. Porto-Foresti. 2010. Identification of hybrids between Neotropical fish Leporinus macrocephalus and Leporinus elongatus by PCR-RFLP and multiplex-PCR: tools for genetic monitoring in aquaculture. Aquaculture, 298: 346-349.

Hashimoto, D. T., F. F. Mendonça, J. A. Senhorini, C. Oliveira, F. Foresti \& F. Porto-Foresti. 2011. Molecular diagnostic methods for identifying Serrasalmid fish (Pacu, Pirapitinga, and Tambaqui) and their hybrids in the Brazilian aquaculture industry. Aquaculture, 321: 49-53.

Hashimoto, D. T., F. D. Prado, J. A. Senhorini, F. Foresti \& F. Porto-Foresti. 2013. Detection of post-F1 fish hybrids in broodstock using molecular markers: approaches for genetic management in aquaculture. Aquaculture Research, 44: 876884.

Hashimoto, D. T., F. D. Prado, J. A. Senhorini, F. Foresti \& F. Porto-Foresti. 2014a. Aquaculture of Neotropical catfish hybrids: genetic strategies for conservation and management. Pp. 1-30. In: Regan, B. (Ed.). Carp and catfish: biology, behavior and conservation strategies. Hauppage, New York, Nova Science Publishers.

Hashimoto, D. T., J. A. Senhorini, F. Foresti, P. Martinez \& F. Porto-Foresti. 2014b. Genetic identification of F1 and post-F1 Serrasalmid juvenile hybrids in Brazilian aquaculture. PLoS ONE, 9: e89902.

Hashimoto, D. T., J. A. Senhorini, F. Foresti \& F. Porto-Foresti. 2012. Interspecific fish hybrids in Brazil: management of genetic resources for sustainable use. Reviews in Aquaculture, 4: 108-118.

IBAMA. 2007. Estatística da pesca 2007: Brasil - Grandes regiões e unidades da Federação. Brasília, DF, 113p.

Mia, M. Y., J. B. Taggart, A. E. Gilmour, A. A. Gheyas, T. K. Das, A. H. M. Kohinoor, M. A. Rahman, M. A. Sattar, M. G. Hussain, M. A. Mazid, D. J. Penman \& B. J. McAndrew. 2005. Detection of hybridization between Chinese carp species (Hypophthalmichthys molitrix and Aristichthys nobilis) in hatchery broodstock in Bangladesh, using DNA microsatellite loci. Aquaculture, 247: 267-273.

Moro, G. V., F. P. Rezende, A. L. Alves, D. T. Hashimoto, E. S. Varela \& L. S. Torati. 2013. Espécies de peixe para piscicultura. 
Pp. 29-70. In: Rodrigues, A. P. O., A. F. Lima, A. L. Alves, D. K. Rosa, L. S. Torati \& V. R. V. Santos (Eds.). Piscicultura de água doce: multiplicando conhecimentos. Brasília, DF, Embrapa.

Moyer, G. R., B. M. Burr \& C. Krajewski. 2004. Phylogenetic relationships of thorny catfishes (Siluriformes: Doradidae) inferred from molecular and morphological data. Zoological Journal of the Linnean Society, 140: 551-575.

Orsi, M. L. \& A. A. Agostinho. 1999. Introdução de espécies de peixes por escapes acidentais de tanques de cultivo em rios da bacia do rio Paraná, Brasil. Revista Brasileira de Zoologia, 16: $557-560$.

Paiva, M. P., M. F. de Andrade-Tubino \& M. P. Godoy. 2002. As represas e os peixes nativos do Rio Grande - Bacia do Paraná - Brasil. Rio de Janeiro, Interciência, 78p.

Palumbi, S. R. 1996. Nucleic acids II: the polymerase chain reaction. Pp. 205-247. In: Hillis, D. M., C. Moritz \& B. K. Mable (Eds.). Molecular systematics. Sunderland, Sinauer.

Porto-Foresti, F., D. T. Hashimoto, F. D. Prado, J. A. Senhorini \& F. Foresti. 2013. Genetic markers for the identification of hybrids among catfish species of the family Pimelodidae. Journal of Applied Ichthyology, 29: 643-647.

Porto-Foresti, F., D. T. Hashimoto, J. A. Senhorini \& F. Foresti. 2010. Hibridação em piscicultura: monitoramento e perspectivas. Pp. 589-606. In: Baldisserotto, B. \& L. C. Gomes (Orgs.). Espécies nativas para a piscicultura no Brasil. Santa Maria, Universidade Federal de Santa Maria.

Prado, F. D., D. T. Hashimoto, F. F. Mendonça, J. A. Senhorini, F. Foresti \& F. Porto-Foresti. 2011. Molecular identification of hybrids between Neotropical catfish species Pseudoplatystoma corruscans and Pseudoplatystoma reticulatum. Aquaculture Research, 42: 1890-1894.
Prado, F. D., D. T. Hashimoto, J. A. Senhorini, F. Foresti \& F. PortoForesti. 2012b. Detection of hybrids and genetic introgression in wild stocks of two catfish species (Siluriformes: Pimelodidae): the impact of hatcheries in Brazil. Fisheries Research, 125-126: 300-305.

Prado, F. D., T. L. Nunes, J. A. Senhorini, J. Bortolozzi, F. Foresti \& F. Porto-Foresti. 2012a. Cytogenetic characterization of F1, F2 and backcross hybrids of the Neotropical catfish species Pseudoplatystoma corruscans and Pseudoplatystoma reticulatum (Pimelodidae, Siluriformes). Genetics and Molecular Biology, 35: 57-64.

Suplicy, F. M. 2007. Freshwater fish seed resources in Brazil. Pp. 129-143. In: Bondad-Reantaso, M. G. (Ed.). Assessment of freshwater fish seed resources for sustainable aquaculture. Rome, FAO (Fisheries Technical Paper, no. 501).

Vaini, J. O., A. B. Grisolia, F. D. Prado \& F. Porto-Foresti. 2014. Genetic identification of interspecific hybrid of Neotropical catfish species (Pseudoplatystoma corruscans vs. Pseudoplatystoma reticulatum) in rivers of Mato Grosso do Sul State, Brazil. Neotropical Ichthyology, 12: 635-641.

White T. J., T. Bruns, S. Lee \& J. Taylor. 1990. Amplification and direct sequencing of fungal ribosomal RNA genes for phylogenetics. Pp. 315-322. In: Innis, M. A., D. H. Gelfand, J. J. Sninsky \& T. J. White (Eds.). PCR Protocols: a guide to methods and applications. San Diego, Academic Press.

Submitted September 15, 2015 Accepted January 24, 2016 by Cláudio Oliveira 